\title{
GLOBAL JURASSIC FLORAS AND CLIMATE
}

Yao, Jiping, Paleogeographic Atlas Project, Department of Geophysical Sciences, University of Chicago, 5734 S. Ellis, Chicago, IL 60637

The present day vegetation types on the earth are to a large extent controlled by climatic conditions that are mainly reflected in variations in moisture availability and temperature through the annual cycle. Therefore, fossil plants can be used as a tool to elucidate both spatial and temporal climatic changes. The global climates of the Jurassic can be interpreted with fossil floras because many parts of world have yielded abundant floral localities. Those records not only provide a strong basis for the qualitative depiction of vegetation patterns, but also provide quantitative data for the reconstruction of paleophytogeographic provinces and climatic changes. The computer program known as "Detrended Correspondence Analysis" written by Hill (1979) was used in this study. The floral data for this quantitative approach was assembled at the generic level mainly from the published literature. By definition, it is a method based on ordination scores, that is, the species ordination scores are averages of the sample ordination scores, and vice versa.

The scatter diagrams of the first two axes of genera and sample sites derived by detrended correspondence analysis were obtained for the intervals of Early, Middle, and Late Jurassic. The gradient variations of both genera and sample sites are reflected by the scores along axis 1 and 2 because axis 1 and axis 2 maximize the correlation of species and sample sites according to this technique. In the ordination score diagrams, the genera in similar climatic environments and the sample sites in similar paleogeographic regions are relatively close to each other along axis 1 and axis 2.

One of characteristics of the Jurassic vegetation is the taxonomic stability through the period compared with other periods of time. Therefore, the generic scores of axis 1 for the Early and Middle, Middle and Late, and Early and Late Jurassic can be plotted against each other. The regression line can be used to assign the scores to the ordered genera. This is the technique used to complement the ordered genera from the gradient score diagrams and to eliminate the effect of some local genera. The genera adjacent to the regression line can be reliably used to reflect climatic gradients. The gradient changes were used to define biomes and to determine phytogeographic patterns on a global scale.

The quantitative analysis clearly shows that the use of floral data can achieve fast and satisfactory results in paleoclimatic studies of the Jurassic. For example, the coolwet temperate climate is reflected by a ginkgophyte dominated deciduous flora, such as Ginkgo, Czekanowskia, Phoenicopsis, and Baiera, whereas the subtropical warm and drier climate is represented by cycad dominated floras, such as Anomozamites, Nilssonia, Pterophylum, Ptilophyllum, Ctenis, and Otozamites. The general conclusion of earlier workers that Jurassic floras were especially uniform through the earth is true in the relative sense only, and the ordination study allows for the subdivision of the rather shallow gradients that do exist. 